\title{
DEVELOPMENT OF OBSTACLE AVOIDANCE TECHNIQUE IN WEB-BASED GEOGRAPHIC INFORMATION SYSTEM FOR TRAFFIC MANAGEMENT USING OPEN SOURCE SOFTWARE
}

\author{
${ }^{1}$ Nik Mohd Ramli Nik Yusoff, ${ }^{1,2}$ Helmi Zulhaidi Mohd Shafri, \\ ${ }^{1}$ Ratnasamy Muniandy and ${ }^{1}$ Islam Mohammad Wali \\ ${ }^{1}$ Department of Civil Engineering, \\ ${ }^{2}$ Geospatial Information Science Research Centre (GISRC), \\ Faculty of Engineering, Universiti Putra Malaysia (UPM), 43400 Serdang, Selangor, Malaysia
}

Received 2013-07-12; Revised 2013-11-25; Accepted 2014-02-22

\begin{abstract}
The shortest path routing is one of the well-known network analysis techniques implemented in road management systems. Pg Routing as an extension of Postgre SQL/Post GIS database is an open source library that implements the Dijkstra shortest path algorithm. However, the functionality to avoid obstacles in that analysis is still limited. Therefore, this study was conducted to enable obstacle avoidance function in the existing pgRouting algorithm using OpenStreetMap road network. By implementing this function, it enhances the Dijkstra algorithm ability in network analysis. In this study a dynamic restriction feature is added at the program level to represent the obstacles on the road. With this modification the algorithm is now able to generate an alternative route by avoiding the existence of obstacles on the roads. By using OpenLayers and PHP a web-based GIS platform was developed to ease the system's usability.
\end{abstract}

Keywords: Road Management, WebGIS, PostgreSQL, PostGIS, pgRouting, Dijkstra Algorithm

\section{INTRODUCTION}

Transportation is the main infrastructure in every country and an important component of national economies. It is used to enable vehicles to travel safely and as quickly as possible. Therefore, the road conditions need to be well maintained so that journey time can be predicted more accurately and minimize the occurrences of road accidents (Kubota et al., 2011).

In developed countries, road management systems have been developed and operational for quite some time. For example, the USA has developed an internet- based system for road maintenance since 1995 (Feng and Quanwen, 2010). Meanwhile, Japan has operated a portal system for road monitoring for their country since 2004 (Kubota et al., 2011). In spite of all the advances being made in this area the current systems can still be expanded in terms of data interoperability and spatial analysis to enhance their usage. Spatial analysis is extremely important in network analysis to assist users in making better decisions. For example, El-Houssaini and Badri (2012) developed a comprehensive system for road accidents using a GIS platform. A spatial analysis technique was applied in the system to find the shortest route based on cost and distance using Dijkstra algorithm. Although the system could show the locations of road accidents on a web, it could not give alternative route to avoid the road accidents location.

GIS has been applied in different areas of transportation since the 1980s (Castro et al., 2011). It is not only focused on road management, it has been applied in several different fields such as facility management system (Bode and Cho, 2010), real-time and off-line routing system (Xinkai et al., 2009; Santos et al., 2011), transit planning engine (Singh and Kumar, 2010), vehicle navigation system (Repoussis et al., 2009), traffic and Corresponding Author: Helmi Zulhaidi Mohd Shafri, Department of Civil Engineering, Faculty of Engineering, Universiti Putra Malaysia(UPM), 43400 Serdang, Selangor, Malaysia 
congestion system (Pei and Jiao, 2011) and highway safety assessment (Davoodi et al., 2010).

In the current decade, Information Technology (IT) has become indispensable. Web-based GIS or WebGIS is the Internet-based platform that provides client-side applications using WWW protocols (Feng and Quanwen, 2010; Xie, 2010). The geographical web can embed spatial and non-spatial data. This technology can reduce the current disadvantages in developing online systems on the internet (Xinkai et al., 2009). WebGIS has many advantages compared to desktop GIS (Xie, 2010). It can be accessed anywhere for data sharing as long as the internet connection is available (Xie, 2010; Kubota et al., 2011; Olfat et al., 2013). Numerous studies have examined the ability of Web-based GIS system in managing road transport data (Deng and Mo, 2009; Feng and Quanwen, 2010; Xie, 2010). This method was carried out by researchers in order to replace traditional method in managing road network that encounter some limitations (Deng and Mo, 2009; Singh and Kumar, 2010).

Generally, WebGIS is extended with special database that can support geographic data. PostgreSQL is a popular database for spatial data. It is an open source database and can be run in almost all operating systems (Firdhous et al., 2010). Other than that, pictures, sounds and videos can be stored in the database. To make it geospatially enabled, PostgreSQL is extended with PostGIS. In order to activate road searching, pgRouting is used in the system. pgRouting is a plugin of postgreSQL/postGIS that has routing capabilities for network analysis (Firdhous et al., 2010). Several studies have utilized pgRouting to calculate the shortest route (Firdhous et al., 2010; García et al., 2013; Goetz and Zipf, 2013). Firdhous et al. (2010) conducted a study to find an optimal path between two locations in urban area. However, there is no function to avoid the obstacles that occur on the road.

The shortest route is calculated by several algorithms that have been developed in the pgRouting application such as Dijkstra algorithm, A-star algorithm and ShootingStar algorithm (Zhang and He, 2012). Some parameters can be added and modified in this application due to the needs. Each algorithm has specific functions and produces different results. Dijkstra algorithm can perform a better response in terms of time for single-source cases (Orlin et al., 2010; Lu and Camitz, 2011). Most studies used this algorithm to perform shortest route because it is widely used in GIS software (El-Houssaini and Badri, 2012) and can provide better result in terms of time response (Firdhous et al., 2010). Thus, this algorithm is used to calculate the distance between two nodes, source and target in this study. However, the functionality to avoid obstacles is still limited (Wang and Zlatanova, 2013). Users can calculate the shortest route using existing applications but they can hardly avoid any obstacle on the road. Therefore, this study was conducted to enable obstacle avoidance function in the existing pgRouting algorithm using OpenStreetMap road network.

The objective of this study is to provide a real-time routing system that is able to run shortest path analysis between two locations and avoid obstacles on the road using pgRouting application. This system will be beneficial for both the public users as they plan their journey to avoid traffic problems and also the highway maintenance administration as they analyse the impact of road blocks during maintenance. Furthermore, this system can be accessed widely as it is internet-based and provides real-time information.

\section{MATERIALS AND METHODS}

The overall flow chart of the system is shown in Fig. 1. It consists of data acquisition, modification of database, development of application, enabling obstacles in the database, testing and comparing the result.

\subsection{Requirements}

This study required open source software in order to minimise the cost. For instance, Minhua (2010) constructed web-based system for transportation modelling platform with low cost investment along with increased processing speed. A number of researches have been carried out to show the benefit of open-source software in web-based GIS system development (Hall et al., 2010; Evans and Sabel, 2012). Furthermore, shortest path analysis has been added in the system to enhance the functionality in network analysis. Figure 2 shows the architecture and software involved in this system.

The user interface is a medium of interaction between user and system. Basically, a web browser is needed to retrieve, present and traverse information from database to the interface for users. Some programming language is needed in designing the interface. In this study, the interface was developed using a combination of HTML and JavaScript programming languages to produce a dynamic and user-friendly interface. Web mapping was required to enable the server to handle and visualize geographic information on web. There are several web mapping applications that can be used such as Google Maps, OpenStreetMap and Microsoft Bing Maps. OpenLayers JavaScript library was used as a web mapping framework because it is flexible and can use both Google Maps and Open Street Map as base maps. 


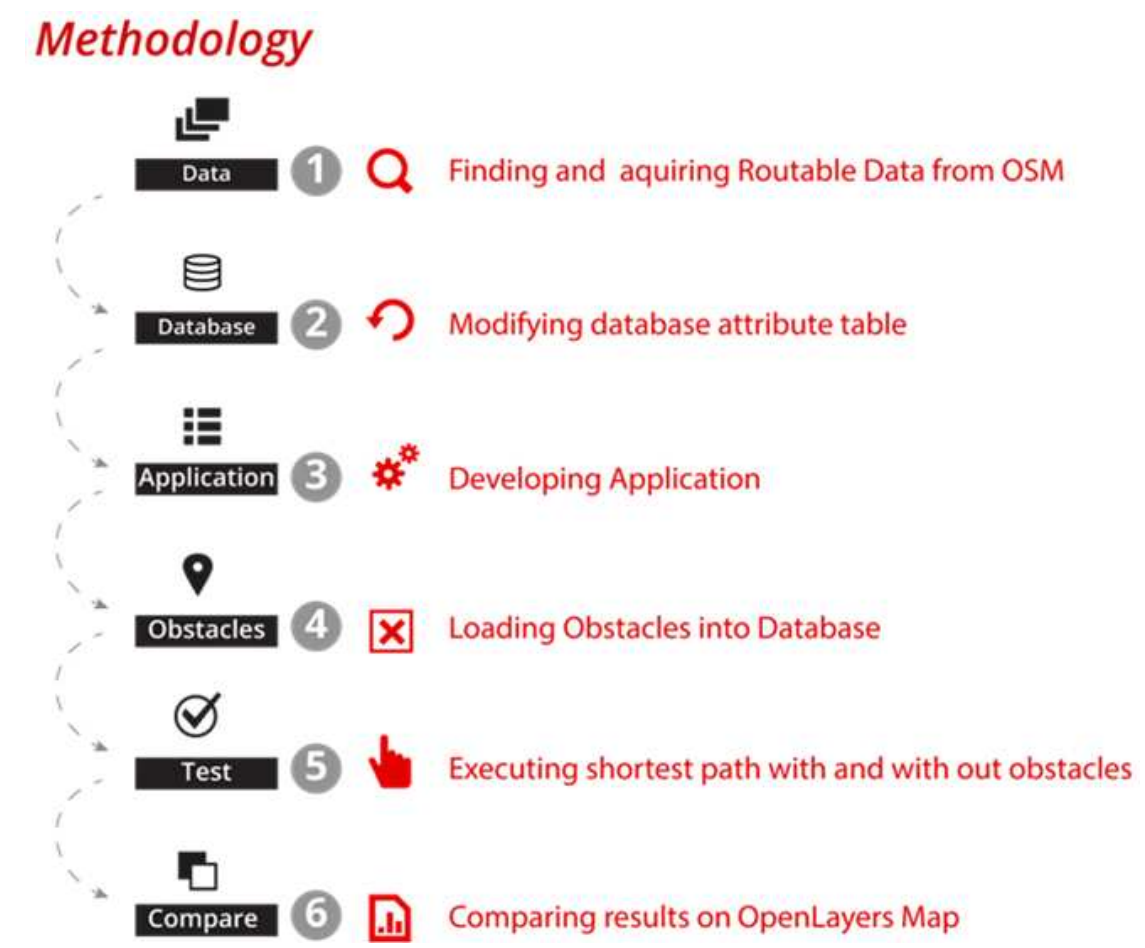

Fig. 1. Overall flow chart of the system

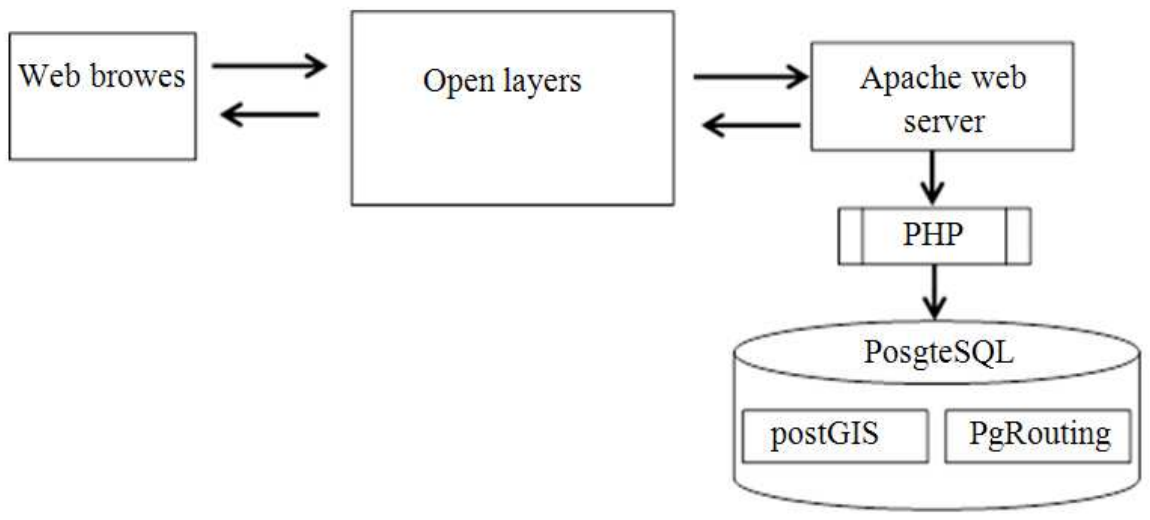

Fig. 2. The architecture and software involved in the system

It also has the ability to visualize road information on the web because it allows users to build dynamic maps on web browser. The functions of OpenLayers can easily be extended because it is an open source web mapping framework and supported by many applications.

A workstation with window 7 operating system was used as the server-side for the system. The hardware specification of the server was a $2.4 \mathrm{GHz}$ Intel® core $^{\mathrm{TM}}$ i5 processor with Random Access Memory (RAM) of 4GB. The server run WampServer to retrieve data from postgreSQL database using the PHP language. The database used was PostgreSQL 9.1 with PostGIS 1.5 spatial extension to support geographic data. pgRouting was also needed to run routing analysis. All the software were installed on one server in order to execute the functions in the system. 


\subsection{System Design}

The area where most important work was done in this study is the system design. First the database was restructured with the addition of a new attribute column and a new table. With the database in place the rest of the system was developed using the database as the driver of the system.

\subsubsection{Development of Database}

Data is one of the main components in GIS analysis. Without data, GIS cannot be run and no information can be displayed. To ensure the data is well maintained a Data Base Management System (DBMS) is required. In order to support spatial datasets in PostgreSQL, PostGIS is required. The coordinates of geometry such as points, lines and polygons are stored in the database and visualized on a map. To conduct this study, the roads data were downloaded from OpenStreetMap website because it was the biggest external data provider for vector road data and easy to handle by free software (Sekimoto et al., 2012). The downloaded data had to be converted into SQL format because OpenStreetMap only provides the data in OSM format. Osm2po was used to convert OSM format to SQL format before the data was stored in postgreSQL database. Osm2po is an engine that can be used as a data converter and routing engine in windows operating system. Afterward the data were stored in postgreSQL and ready to be used with postGIS and pgRouting.

A table was created to ease the process of management and it was named as road network. Several attributes were provided in the table such as id, osm name, source, target, clazz, flags, km, kmh, $\mathrm{x} 1, \mathrm{y} 1, \mathrm{x} 2$, y2, geom way, cost. These attributes give the characteristic of the road which is called attribute data. When the shortest path is generated, the result can be shown in two ways; spatial visualization or attribute table:

$$
\begin{aligned}
& \text { Distance }(D)=\sqrt{\left(X_{2}-X_{1}\right)^{2}+\left(Y_{2}-Y_{1}\right)^{2}} \\
& \text { Total Distance, } D_{n}=D_{1}+D_{2}+D_{3}+\ldots D_{n}
\end{aligned}
$$

To enable the detection of obstructions on the road, the attribute for restriction was added in "road network" table. The attribute was named as "obstruction". A value of an integer greater than 0 indicates the presence of an obstruction at the given node and a value of 0 indicates absence of obstruction at the node. When obstacle exists, the ID of the obstruction was stored in the "obstruction" column. Another table was created to represent obstruction table which stored information such as obstruction ID, obstruction category, date start, date end, status and description. These two tables were linked using Node ID column as a foreign key.

\subsection{System Implementation}

To provide a successful road management system, pgRouting application is required to conduct shortest path by implementing Dijkstra algorithm.

\subsection{1. pgRouting Application and Dijkstra Algorithm}

pgRouting also known as PgDijkstra is an application used with PostgreSQL/PostGIS to calculate the shortest route between two nodes using Dijkstra algorithm. After gradual optimization, A-star and Shooting-star algorithms were added in pgRouting. It became a core of the shortest path search because the attribute data are dynamic and can be modified by many open source clients (Zhang and He, 2012).

In literature reviews, Dijkstra algorithm is mostly used to search the shortest route between two nodes. This algorithm has the main function of searching the shortest distance between adjacent points. Figure 3 represents the concept to calculate distance between two nodes. Node $\mathrm{A}\left(\mathrm{X}_{1}, \mathrm{Y}_{1}\right)$ represents the starting point while node $\mathrm{B}\left(\mathrm{X}_{2}, \mathrm{Y}_{2}\right)$ represents the ending point. Equation 1 shows how to find the distance between these two points.

Figure 4a shows how the Dijkstra indicates the shortest path in diagram format. The node $\mathrm{S}$ represents the source while node $\mathrm{T}$ represents the target point. Meanwhile, D represents the distance between adjacent points. There are four possible routes between $\mathrm{S}$ to $\mathrm{T}$ which are SQUT, SQPT, SRPT and SRPQUT. First, Equation 1 was used to calculate distance between SR and SQ. The result shows that the distance between SQ shorter than SR. Therefore, node Q was selected as the path as in Fig. $\mathbf{4 b}$.

Next, the distance between the adjacent nodes of Q is calculated. Figure $4 c$ shows that QU was selected for forward node. Lastly, node $U$ is directly connected to node $\mathrm{T}$ because there is no choice to node $\mathrm{T}$ as in Fig. 4d. Subsequently, Equation 2 was used to calculate the total distance between $\mathrm{S}$ to $\mathrm{T}$ where D-n represents the total distance from start point to destination. Therefore, SQUT route was chosen as the shortest route. 
Nik Mohd Ramli Nik Yusoff et al. / Journal of Computer Science 10 (7): 1259-1268, 2014

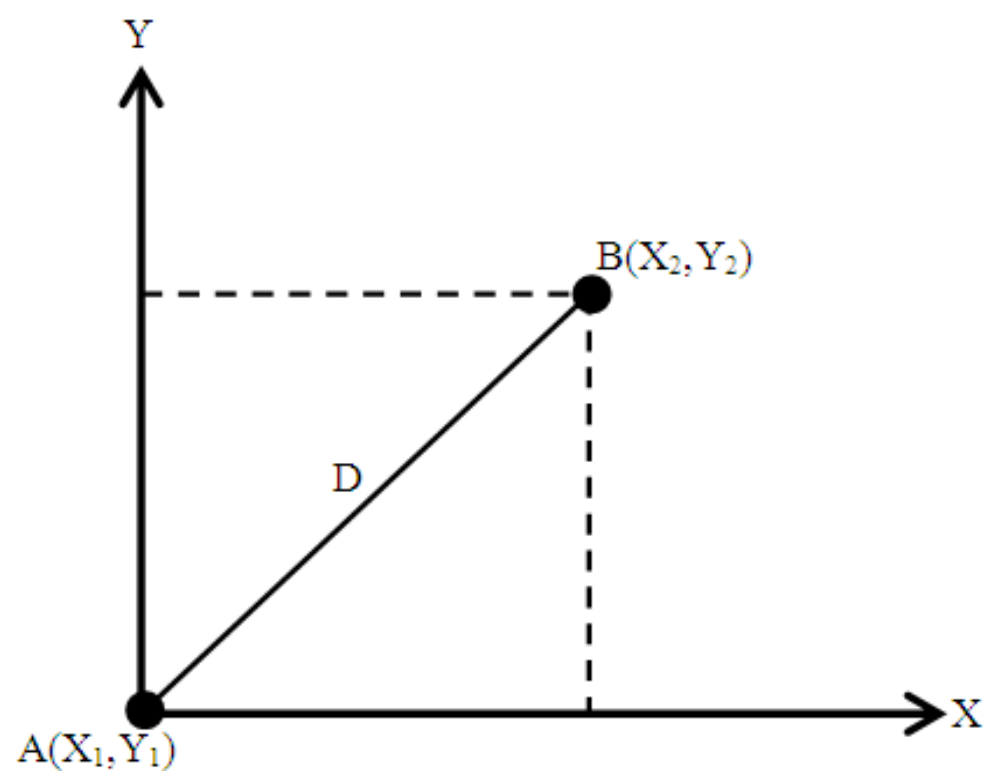

Fig. 3. 2D differential for node A and B

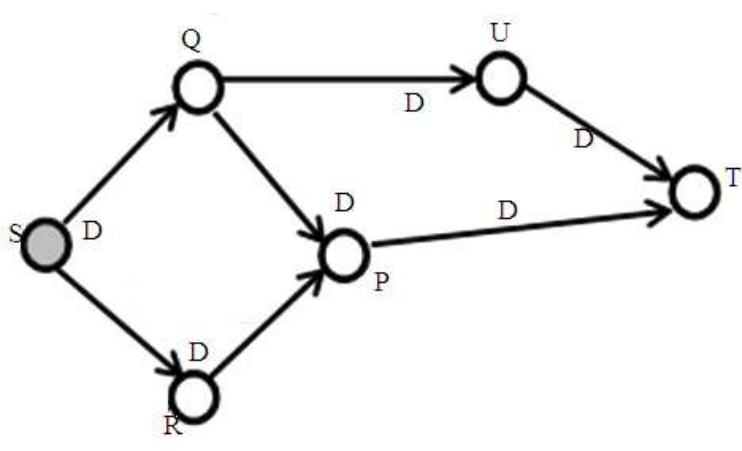

(a)

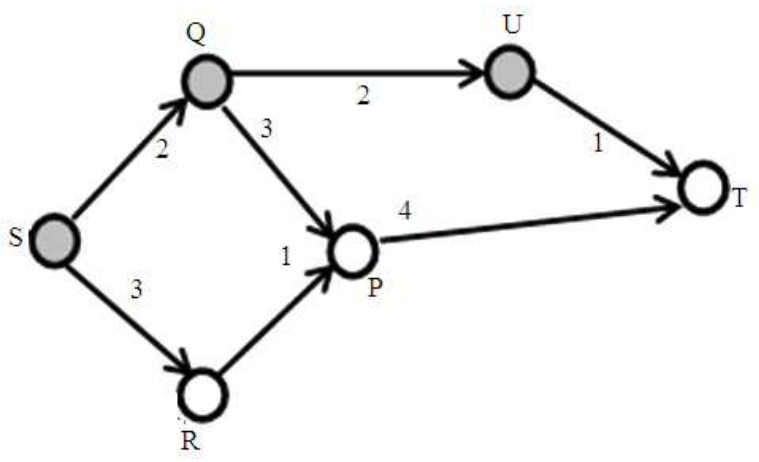

(c)

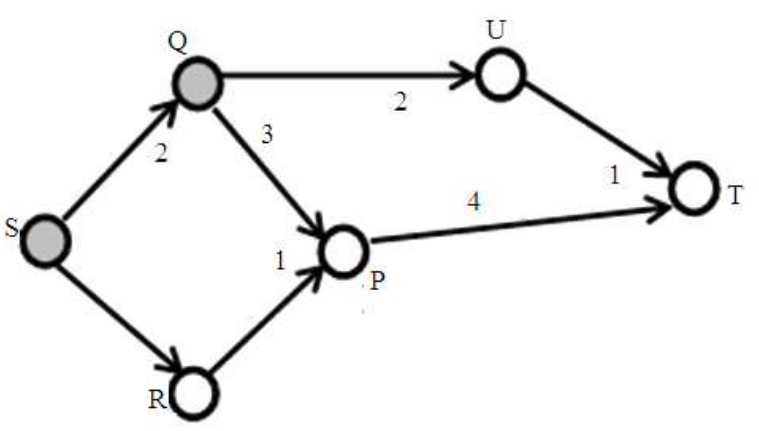

(b)

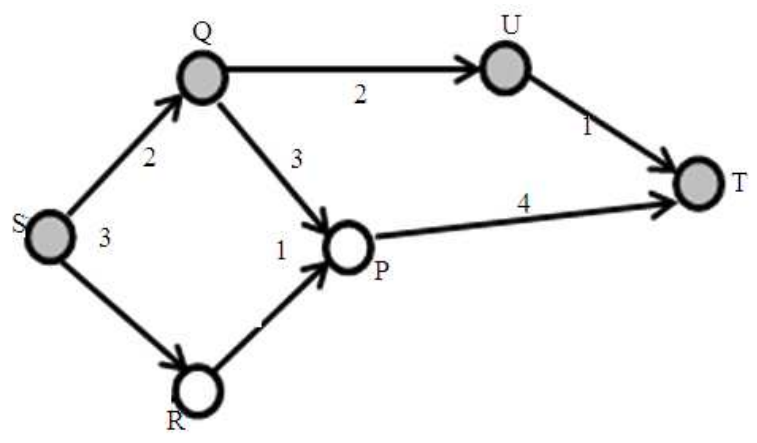

(d)

Fig. 4. Simulation of Dijkstra algorithm in shortest path analysis 


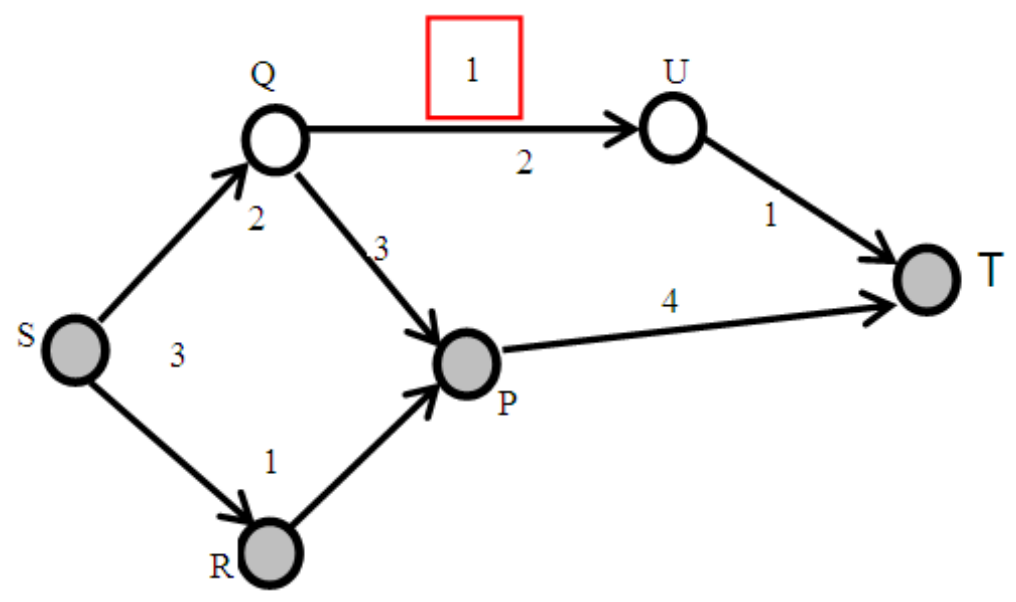

Fig. 5. The existence of obstacle between node $\mathrm{Q}$ and $\mathrm{U}$

Sometimes, the shortest road is not the fastest road. It may have some obstacles such as traffic jam, accidents, roadwork maintenance, road closure and etc. this situation can take more time to arrive at target location. To enable obstacles in this algorithm, a weightage for restriction is added in this function. This weightage represents the events that occur on the road and blocked the users from passing that road. Figure 5 shows the obstacle between node Q and U. During the route searching, the algorithm cannot pass QU route. Thus, the route was diverted to another alternative route. In this situation, SRPT was chosen as the shortest distance and can give less time to arrive at the point $\mathrm{T}$. In addition, users can predict what time they can arrive at their destination.

\section{RESULTS}

The proposed system concentrates on the road administration activities by collecting important information about the condition and status of the road from various sources such as road accidents, schedule of road maintenances, temporary road blocks and traffic flow data. By keeping track of this information, the system can visually highlight road segments based on their conditions as well as geographically visualize the locations of obstacle/restriction events.

\subsection{Shortest Route Analysis}

The shortest path function provided by pgRouting uses the cost of road segments to determine paths to avoid. One simple and primitive way of implementing a cost avoidance function is to specify very high cost values for road segments that are to be avoided in calculating the shortest path. In the proposed system the obstacle avoidance was done across several dimensions-time, road status, events, road maintenance and road accidents.

Figure 6 shows a simple interface that was developed to visualize the routing component of the system. The interface consists of a base map and form fields that capture user input such as the origin node, destination node, as well as date and time. OpenStreetMap was embedded as the base map because it has the capability to extent the function and not depend to any services as Google Maps API. Users can search the shortest route based on origin and destination and display result on map.

The system worked consecutively as in Fig. 7. When users insert the source and target nodes into origin and destination columns respectively, the system will query the input nodes in the database using SQL language. The system then sends the query to the postgreSQL database for processing. At the same time, the computation of shortest path is calculated using the extended pgRouting in the database. Finally, the shortest route result is shown with highlighted colour on the web map.

\subsection{Visualize an Obstacle and Alternative Route Unit}

Although that path is the shortest path, sometimes it has some obstacles along that path. This system provides a function to avoid obstacles on the road. An entry in the database signals a restriction and blocks the road. This causes the system to generate an alternative route to the destination. 


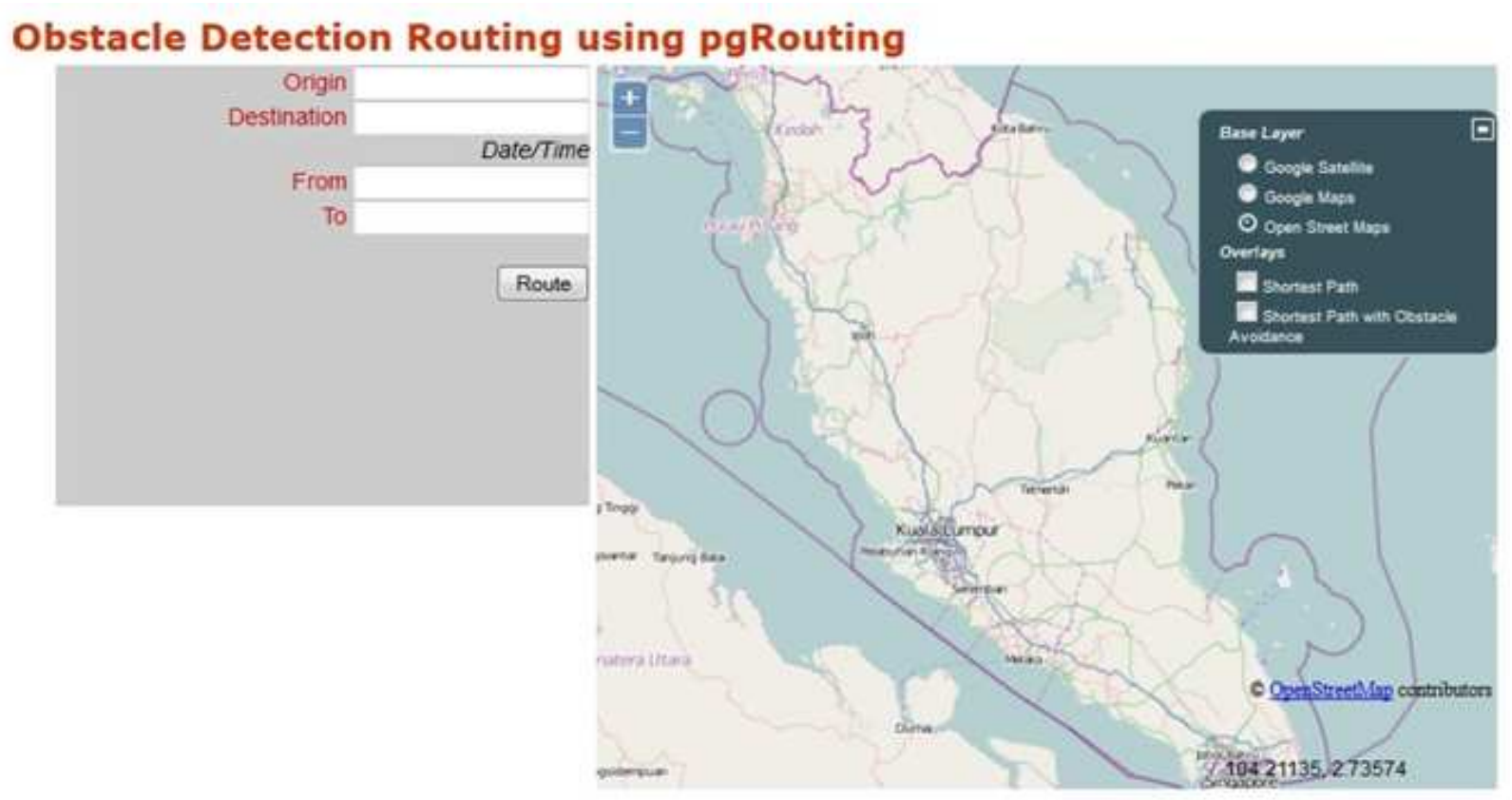

Fig. 6. Main interface for obstacle avoidance

\section{Obstacle Detection Process}

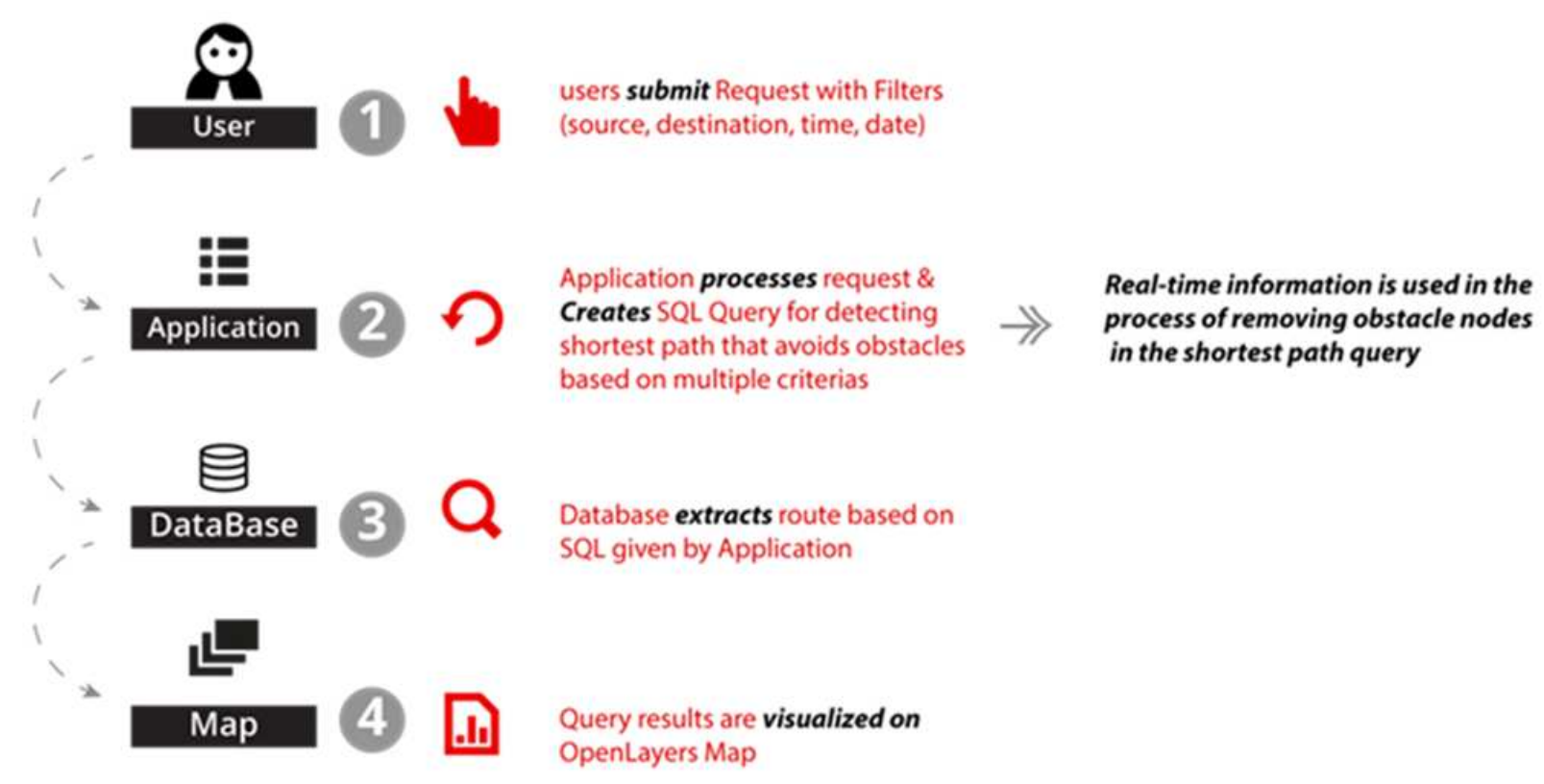

Fig. 7. The work sequence in the system 


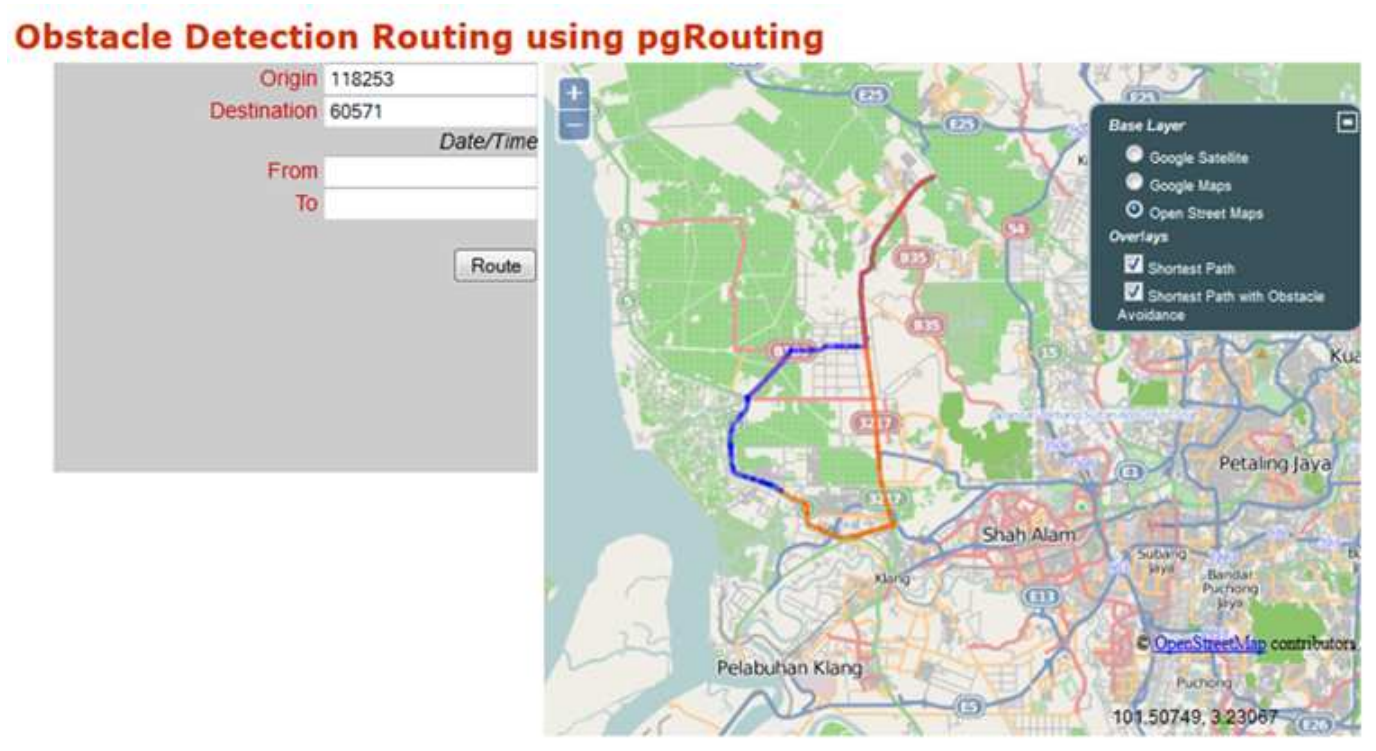

Fig. 8. Obstacle avoidance in shortest path analysis

Figure 8 represents the obstacle avoidance on the road in the system. The highlighted road in blue colour is the shortest route from origin to the destination. Because of the existence of an obstacle, the system generated an alternative route which is highlighted in brown colour. Therefore, users can find alternative route without any obstacle along their way.

\section{DISCUSSION}

This study discusses the development of a web mapping application based on a WebGIS system that will implemente in a road management system. The downloaded data from OpenStreetMap website consisted of information on roads around Peninsular Malaysia. This data was used in this study because it is supported in postgreSQL/postGIS database. To enrich the database, some information has been added such as obstruction information, road accidents, road maintenance schedule and mandatory road closure.

Since the data was downloaded from OpenStreetMap website, it can only give the source and target point in ID format. Therefore, this system can still be improved by using the reverse geocoding to make it more user friendly.

Dijkstra algorithm is a straight forward algorithm for calculating the distance between two nodes and is the most efficient algorithm for calculating the shortest path in road network analysis using the pgRouting extension. Even though it can give the shortest distance in route calculation, it cannot detect the existence of obstacles along the road. A weightage for obstacles on roads was added to avoid the blocked roads. Therefore, this system has the capability to avoid obstacles or restrictions on the road.

This system can work best for road users. They can organize their journey in terms of searching the shortest route. For organizations, this system can be used to monitor all road constructions occurring on the road. All information is gathered in one database so that it is easy to be retrieved and displayed on the interface. In order to make this system cost-effective, open-source software was used which is becoming a new trend in system development.

\section{CONCLUSION}

A web-based GIS system for traffic management activity using open-source software was developed in this study. By accessing the web, users can easily visualize the traffic condition anywhere in the world either on a desktop or mobile device. As it is opensource development, the system can be modified freely without any restriction from software developer. Due to the fact that the data was downloaded from OpenStreetMap website, it has some limitations in 
terms of data quality. For future research, we suggest that the data are collected from transportation authorities to improve the data quality. It is hoped that this research will inspire others to create more complex obstacles such as time based restrictions using the presented method. Another area for future study is to improve the quality of visualization, for example displaying obstacles in multiple dimensions.

\section{ACKNOWLEDGEMENT}

The researchers thank Universiti Putra Malaysia (UPM) for providing the research grant under the Research University Grant Scheme (RUGS) and UPM for providing graduate scholarship in completing the research. We also like to thank our anonymous reviewers whose comments helped to improve this study.

\section{REFERENCES}

Bode, T. and Y.K. Cho, 2010. Framework for web-based highway construction geospatial data management. Proceedings of the Construction Research Congress, American Society of Civil Engineers, May 8-10, IEEE Xplore Press, Alberta, Canada, pp: 607-616. DOI: 10.1061/41109(373)61

Castro, M., L. Iglesias, J.A. Sánchez, and L. Ambrosio, 2011. Sight distance analysis of highways using GIS tools. Transport. Res. C, 19: 997-1005. DOI: 10.1016/j.trc.2011.05.012

Davoodi, S.R., H. Hamid, S. Arintono and R. Muniandy, 2010. Motorcycle characteristics for sight distance investigation on exclusive motorcycle lanes. J. Transport. Eng., 137: 492-495. DOI: 10.1061/(ASCE)TE.1943-5436.0000226

Deng, J. and Y. Mo, 2009. Intelligent decision support system for road network planning. Proceedings of the International Colloquium of Computing, Communication, Control and Management, Aug. 89, IEEE Xplore Press, Sanya, pp: 139-142. DOI: 10.1109/CCCM.2009.5267809

El-Houssaini, S. and A. Badri, 2012. A web-based spatial decision support system for effective monitoring and routing problem. Proceedings of the International Conference of Multimedia Computing and Systems, May 10-12, IEEE Xplore Press, Tangier, pp: 669674. DOI: 10.1109/ICMCS.2012.6320116

Evans, B., and C.E. Sabel, 2012. Open-Source webbased geographical information system for health exposure assessment. Int. J. Health Geograph., 11: 2-2. DOI: $10.1186 / 1476-072 X-11-2$
Feng, X. and L. Quanwen, 2010. Development of highway management system based webgis. Proceedings of the WASE International Conference of Information Engineering, Aug. 14-15, IEEE Xplore Press, Beidaihe, pp: 136-138. DOI: 10.1109/ICIE.2010.323

Firdhous, M.F.M., D.L Basnayake, K.H.L. Kodithuwakku, N.K. Hatthalla and N.W. Charlin et al., 2010. Routeadvising in a dynamic environmenta high-tech approach. Innovations in Computing Sciences and Software Engineering. Springer, Netherlands, pp: 249-254, DOI: 10.1007/978-90481-9112-3-42

García, K., S. Mendoza, D. Decouchant, J. Rodríguez and T. Pérez, 2013. Determining and locating the closest available resources to mobile collaborators. Expert, Syst. Applic., 40: 2511-2529. DOI: 10.1016/j.eswa.2012.10.069

Goetz, M. and A. Zipf, 2013. Indoor Route Planning with Volunteered Geographic Information on a (Mobile) Web-Based Platform. In: Progress in Location-Based Services, Krisp, J.M. (Ed.), Springer Berlin Heidelberg, ISBN: 9783642342028, pp: 211231.

Hall, G.B., R. Chipeniuk, R.D. Feick, M.G. Leahy and V. Deparday, 2010. Community-based production of geographic information using open source software and Web 2.0. Int. J. Geograph. Inform. Sci., 24: 761781. DOI: $10.1080 / 13658810903213288$

Kubota, S., T. Sugawara, T. Kosawada and A. Abe, 2011. Web GIS-bsed information portal system for road maintenance. Proceedings of the 19th International Conference of Geoinformatics, June 24-26. IEEE Xplore Press, Shanghai, pp: 1-5. DOI: 10.1109/GeoInformatics.2011.5981022

Lu, X., and M. Camitz, 2011. Finding the shortest paths by node combination. Applied Math. Comput., 217: 6401-6408. DOI: 10.1016/j.amc.2011.01.019

Minhua, W., 2010. A new way of transportation modeling-cloud computing based internet solution for transportation modeling. Proceedings of the 10th International Conference of Chinese Transportation Professionals, American Society of Civil Engineers, Aug. 4-8, IEEE Xplore Press, Beijing, pp: 10861096. DOI: $10.1061 / 41127(382) 116$

Olfat, H., M. Kalantari, A. Rajabifard, H. Senot, and I.P. Williamson, 2013. A GML-based approach to automate spatial metadata updating. Int. J. Geograph. Inform. Sci., 27: 231-250. DOI: $10.1080 / 13658816.2012 .678853$ 
Orlin, J.B., K. Madduri, K. Subramani, and M. Williamson, 2010. A faster algorithm for the single source shortest path problem with few distinct positive lengths. J. Discrete Algorithms, 8: 189-198. DOI: 10.1016/j.jda.2009.03.001

Pei, Y. and G. Jiao, 2011. Analysis of public traffic information system based on WebGIS. Proceedings of the 6th IEEE Join International Conference of Information Technology and Artificial Intelligence, Aug. 20-22, IEEE Xplore Press, Chongqing, pp: 448-450. DOI: 10.1109/ITAIC.2011.6030244

Repoussis, P.P., D.C. Paraskevopoulos, G. Zobolas, C.D. Tarantilis, and G. Ioannou, 2009. A web-based decision support system for waste lube oils collection and recycling. Eur. J. Operat. Res., 195: 676-700. DOI: 10.1016/j.ejor.2007.11.004

Santos, L., J. Coutinho-Rodrigues and C.H. Antunes, 2011. A web spatial decision support system for vehicle routing using Google Maps. Decision, Support, Syst., 51: 1-9. DOI: 10.1016/j.dss.2010.11.008

Sekimoto, Y., A. Watanabe, T. Nakamura, H. Kanasugi and T. Usui, 2012. Combination of spatio-temporal correction methods using traffic survey data for reconstruction of people flow. Pervasive Mobile Comput. DOI: 10.1016/j.pmcj.2012.10.005
Singh, V., and P. Kumar, 2010. Web-based advanced traveler information system for developing countries. J. Transport. Eng., 136: 836-845. DOI: 10.1061/(ASCE)TE.1943-5436.0000144

Wang, Z., and S. Zlatanova, 2013. Taxonomy of navigation for first responders. In: Progress in Location-Based Services, Krisp, J.M. (Ed.)., Lecture Notes in Geoinformation and Cartography, New York Dordrecht London, ISBN: 978-3-642-34203-5, pp: 297-315.

Xie, F., 2010. Design and implementation of highway management system based WebGIS. J. Net., 5: 1389-1392: $\quad 1796-2056 . \quad$ DOI: 10.4304/jnw.5.12.1389-1392

Xinkai, Y., S. Jie, Y. Fengtao, D. Yongfeng and D. Zhenchao, 2009. Study of intelligent public transport monitoring system based on WebGIS. Proceedings of the 2nd International Conference of Intelligent Networks and Intelligent Systems, Nov. 1-3, IEEE Xplore Press, Tianjin, pp: 78-81. DOI: 10.1109/ICINIS.2009.29

Zhang, L. and X. He, 2012. Route Search Base on pgRouting. In: Software Engineering and Knowledge Engineering: Theory and Practice, Wu, Y. (Ed.)., Springer, Berlin, ISBN-10: 3642037178, pp: 1003-1007. 